\title{
Rationalization and Reflection Differentially Modulate Prior Attitudes Toward the Purity Domain
}

\author{
Ivar R. Hannikainen and Alejandro Rosas ${ }^{1}$
}

\begin{abstract}
Outside Western, predominantly secular-liberal environments, norms restricting bodily and sexual conduct are widespread. Moralization in the socalled purity domain has been treated as evidence that some putative violations are victimless. However, respondents themselves disagree: They often report that private yet indecent acts incur self-harm, or harm to one's family and the wider community—a result which we replicate in Study 1 . We then distinguish two cognitive processes that could generate a link between harmfulness and immorality, and recreate them in Studies 2 and 3 : Colombian and British participants were randomly assigned to either reflect (decide whether acts are harmful and reconsider their initial moral judgments) or rationalize (decide whether acts are immoral and reconsider their initial harmfulness beliefs). In both countries, reflection promoted opposition to unjust, but not impure, behavior. Additionally, in both countries, ruminating on the moral status of impure acts elevated beliefs in the acts' harmfulness. We conclude by suggesting that rationalization aggravates, while reflection mitigates, intergroup disagreement regarding putative violations of purity and decency.
\end{abstract}

\section{Introduction}

Hindu tradition dictates that widows must adhere to brahmacharya, a strict celibate lifestyle, which includes foregoing garlic and a long list other foods believed to cause sexual arousal (Shweder, Mahapatra \& Miller, 1987; Sivananda, 1934). To those from Western, educated, industrialized, rich and democratic (WEIRD for short; see Henrich, Heine \& Norenzayan, 2001) backgrounds, the garlic taboo likely sounds baffling and puritanical: Widows' diets are simply not a moral matter; there is no harm done if widows eat garlic or aphrodisiacs of any kind (Haidt \& Hersh, 2001). More likely, the norm itself is immoral for infringing upon their autonomy.

\footnotetext{
1 Author Note: Ivar R. Hannikainen, Department of Law, Pontifical Catholic University of Rio de Janeiro; Alejandro Rosas, Department of Philosophy, Universidad Nacional de Colombia (National University of Colombia). Correspondence concerning this article should be addressed to Ivar R. Hannikainen, Department of Law, Rua Marquês de São Vicente, 225, Gávea, Rio de Janeiro, Brazil, 22451-900. Contact: ivar.hannikainen@gmail.com
} 
An influential experimental paradigm, credited to Haidt and colleagues (1993), promoted the conclusion that-outside Western, predominantly secular-liberal societies-a range of sexual and culinary choices are moralized even when they are known not to cause any harm. In a famous example, participants were asked whether it would be wrong for someone to eat their deceased pet, assuming it were perfectly harmless to do so. Indeed, participants-particularly those in lower socioeconomic strata-reported that it would be universally wrong and punishable (Haidt et al., 1993). Later studies also demonstrated that disagreements about the permissibility of these acts offer insight into discord in the real world-i.e., between religious conservatives and secular liberals (Inbar, Pizarro \& Bloom, 2009; Piazza \& Landy, 2013).

The discovery that moral codes around the world abound with purity and decency norms revolutionized the science of moral cognition (Haidt, 2007; Nichols, 2004), overturning previously dominant theories according to which harm and suffering form the building blocks of the moral domain (Blair, 1995; Turiel, 1983).

Yet, moral psychologists may have been rash to assume that what in their own eyes (and from a secular-liberal perspective more generally) appears innocuous is perceived that way by their research participants (but see Gray, Schein, \& Ward, 2014). It would seem otherwise: Immersing in the worldviews that sustain purity-related taboos reveals not simply different moral norms, but also a different understanding about matters of fact. For instance, the dietary prohibitions in brahmacharya serve allegedly to protect the deceased husband's spirit, believed to live on at one with his wife eternally. However far-fetched this belief sounds from a secular perspective, it raises the possibility that the 'victimless crimes' literature doesn't undermine the centrality of harm as much as it highlights the more mundane fact that cultures vary in their beliefs about the scope and proper objects of harm (Schein \& Gray, 2015).

The purpose of the present work is to call this assumption into question. We revisit the influential Haidt and colleagues' (1993) study, while posing a new set of questions: Is it harmful to privately eat your dog, French-kiss your sibling, or wipe down the toilet with the national flag? And, if so, are these acts impermissible because they're considered harmful or, rather, described as harmful because they're judged to be impermissible? Below we outline these contrasting causal models and summarize past research documenting their impact upon moral cognition.

\subsection{Reflection: harmfulness $\rightarrow$ immorality}

Seminal work has traced the condemnation of impure acts to heightened disgust sensitivity (Haidt et al., 1993; Haidt \& Hersh, 2001; Inbar et al., 2009; Lieberman \& Lobel, 2012), which in turn may explain the emergence of intergroup moral disagreement-i.e., between religious conservatives and secular liberals (Inbar et al., 2012; Hannikainen, Miller \& Cushman, 2017).

However, when statistically controlling for harmfulness beliefs, belief in harmful outcomes - and not disgust—better predicts moral condemnation (Gray \& Schein, 2016). Furthermore, mediation analyses suggest that beliefs in harmfulness mediate the relationship between disgust sensitivity and moralization (Schein, Ritter \& Gray, 2016). Thus, intergroup moral disagreement may arise from contradictory beliefs about whether impure acts are harmful, and it is these beliefs that proximately drive moral condemnation. 
We refer to this process, whereby an action's harmfulness results in moral attitude change, as reflection. Reflection resembles the way most applied moral reasoning is thought to proceed: e.g., you learn that plastic pollution hurts marine life and, as a result, you come to judge that single-use plastics are morally wrong and/or should be forbidden.

Yet, evidence of reflection in the moralization of purity derives primarily from correlational studies, while convergent experimental evidence is wanting. To evaluate the impact of reflection on moral attitudes in the purity domain, in Studies 2 and 3, we randomly assign some participants to reflect on whether a set of impure and indecent acts are harmful, while recording the effects of reflection on their moral judgments.

\subsection{Rationalization: immorality $\rightarrow$ harmfulness}

A correlation between moral attitudes and beliefs about harm may also emerge if moralization elevates the perception of harm. As individuals spontaneously reflect on a behavior's moral status, they may be motivated to impute harm (Gray et al., 2014; Haidt, 2001; Royzman, Kim \& Leeman, 2015) or align their beliefs about the behavior's harmfulness with their moral evaluations (Liu \& Ditto, 2013). For instance, someone could query their moral attitude towards abortion and, if they feel strongly opposed, thereby embrace the belief that a fetus is already sentient at conception. We refer to this second causal process, by which assessing an action's moral status shapes our harmfulness beliefs, as rationalization.

For instance, Liu and Ditto (2013) randomly assigned participants to read an essay either for or against capital punishment, and observed that participants' beliefs about whether capital punishment harms or helps society shifted in consonance. This occurred even though the essays advanced deontological moral claims (e.g., "It is wrong to solve violence with further violence" vs. "premeditated murders are subhuman and undeserving of mercy") and did not allude to the consequences of capital punishment. This work suggests that a causal arrow may also point in the opposite direction, from evaluation to belief (see also Haidt, 2001; Ditto, Pizarro \& Tannenbaum, 2009; Mercier \& Sperber, 2011).

In our experiments, we make use of slightly distinct methods. Instead of manipulating the target behavior's moral status, we invite participants to freely ponder whether the target behavior "should be allowed or forbidden" (Study 2) and "is morally wrong" (Study 3). We then investigate whether ruminating on the moral status of impure acts promotes the belief that they are harmful.

\subsection{Overview}

Past research has shown that moral disapproval of taboo behavior correlates with the belief in its harmful outcomes (Schein et al., 2016) - a result which we replicate in Study 1. Then, in Studies 2 and 3, we randomly assign participants to engage in either reflection or rationalization-two cognitive processes that could give rise to the aforementioned correlation-and record their effects (see Table 2).

Since attitudes towards the purity domain have been found to vary across cultures (Haidt et al., 1993), we draw on both United Kingdom $(n=623)$ and Colombian (i.e., $n=860$ ) respondents-characteristically individualistic and collectivistic societies 
respectively (see Hofstede, Hofstede, \& Minkov, 2010). All study materials, data and code can be found at https://osf.io/fp5t7/.

\section{Study 1}

\subsection{Methods}

\subsubsection{Participants}

183 volunteers (119 women, $\left.M_{\text {age }}=24.3\right)$ were approached on a university campus in Colombia.

\subsubsection{Procedure}

After providing informed consent, participants viewed four scenarios in a fixed order involving taboo behaviors adapted and translated from Haidt and colleagues' (1993) study: Flag (cutting up the national flag and using it to clean the toilet), Promise (breaking a promise to one's deceased mother), Chicken (having sexual intercourse with a whole chicken before cooking it) and Incest (French-kissing one's sibling in private). For each scenario, participants evaluated whether the behavior in question harms the agent herself and/or third-parties, on independent seven-point scales from 0: "no harm at all', to 6: 'very much harm'.

In addition, participants' moral attitudes were recorded using two dichotomous measures, also drawn from Haidt and colleagues' (1993) study: moralization ("Should this private act be prevented and/or punished?"; 1: "Yes", 0: "No"), and objectivism ("Imagine you discover that in country $\mathrm{X}$ people often engage in this act privately, while in country Y they do not. What would you believe?"; 1: "One culture is wrong", 0: "Both cultures are acceptable").

Colombian law (Law 142 of 7/1994) enables municipalities to classify their residents into six socioeconomic strata, primarily for administrative purposes. In our experiment, we draw on this classification scheme in order to replicate Haidt and colleagues' (1993) finding that low-SES people are more opposed to putative violations of the purity and sanctity domain.

\subsubsection{Analysis and predictions}

First, for each harmfulness judgment separately, we conducted a mixed-effects linear regression with crossed random effects of participant and scenario, and reported the model intercept, $a$, as the estimate of mean harmfulness. In a follow-up analysis, we added social stratum as a fixed effect to examine its relationship with harmfulness beliefs.

Then, we turned to our dependent measures: i.e., moralization and objectivism. For each dependent measure, we conducted mixed-effects logistic regression entering social stratum and harmfulness judgments as fixed effects, as well as crossed random effects of participant and scenario.

\subsection{Results}

Looking across scenarios, harmfulness beliefs $\left(\alpha_{\text {Self }}=1.34,95 \%\right.$ CI $[0.61,2.07], \alpha_{\text {Third-party }}$ $=1.19,95 \%$ CI $[0.50,1.88]$ ) were significantly above 'no harm at all', $t$ Self $=4.00, t$ Third-party 
$=3.76, \mathrm{ps}<.001$. The baseline probability of ascribing at least 'some harm' to the self was $36 \%, 95 \%$ CI $[16 \%, 63 \%]$ and $29 \%, 95 \%$ CI $[15 \%, 49 \%]$ to third parties.

As predicted, social stratum was negatively associated with harmfulness beliefs, $\beta_{\text {Self }}=-0.26,95 \%$ CI $[-0.45,-0.07], t=-2.74, p=.007 ; \beta$ Third-party $=-0.20,95 \%$ CI [-0.38, $0.02], t=-2.19, p=.030$.

$\diamond$ Self $\diamond$ Third-party

$\bigcirc$ Objectivism $\bigcirc$ Moralization
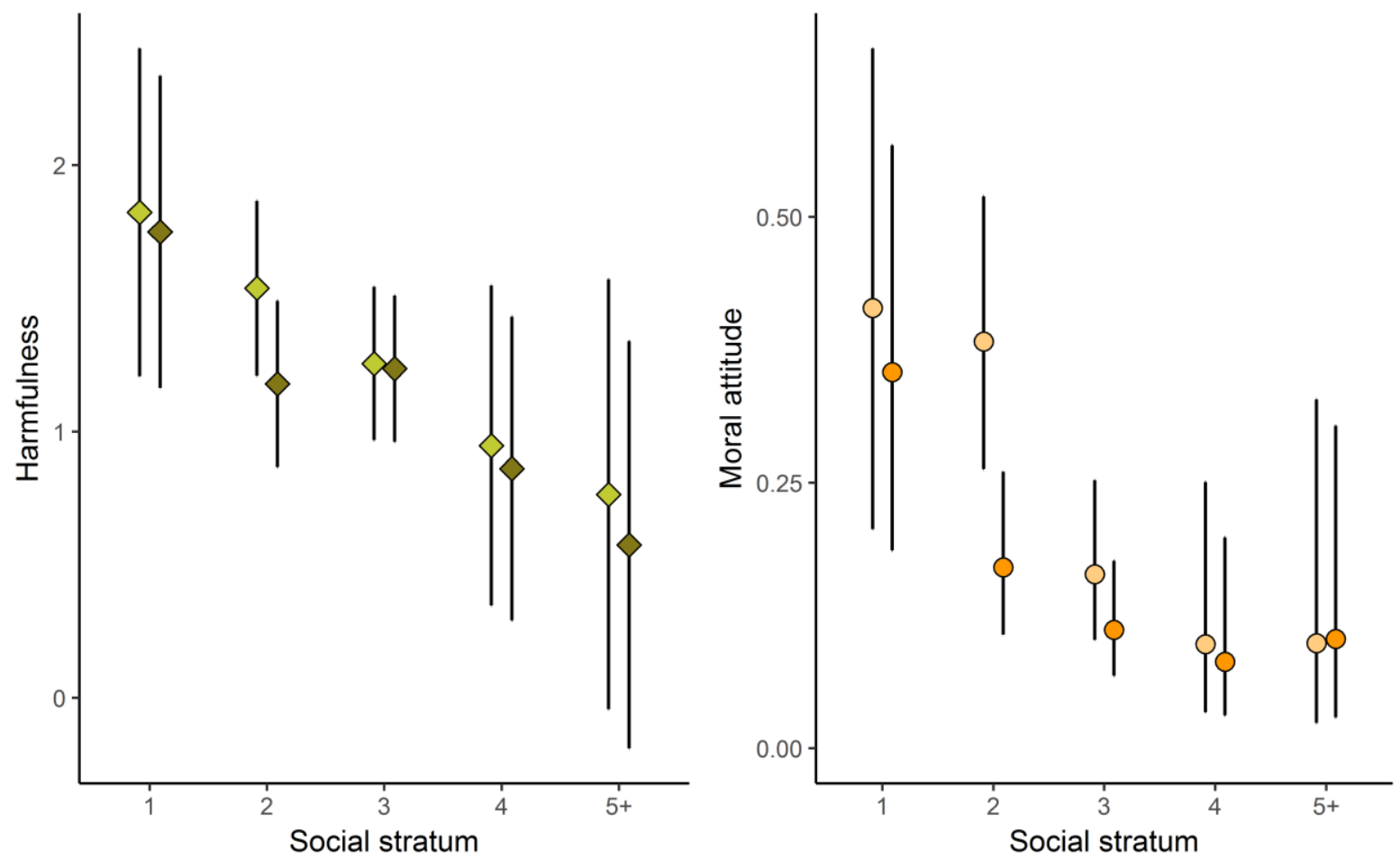

Figure 1. Harm beliefs (left) and moral attitudes (right) by social stratum.

Replicating Haidt and colleagues (1993), social stratum negatively predicted moralization, $O R=0.65,95 \%$ CI $[0.48,0.85], z=-3.01, p=.003$, and objectivism, $O R=$ 0.54, 95\% CI [0.38, 0.73], $z=-3.80, p<.001$ (see Fig. 1).

Finally, controlling for differences across social strata, harm ascriptions were linked to moralization, $O R_{\text {Self }}=1.46,95 \%$ CI $[1.29,1.68], z=5.72, O R_{\text {Third-party }}=1.53$, $95 \%$ CI $[1.34,1.77], z=5.91$, as well as objectivism, $O R_{\text {Self }}=1.63,95 \%$ CI $[1.41,1.89], z=$ $6.54, O R_{\text {Third-party }}=1.35,95 \%$ CI $[1.18,1.57], z=4.16$, all $p$ s $<.001$.

\subsection{Discussion}

Though many participants viewed purity-domain violations as harmless, some participants-especially from lower socioeconomic strata-reported that purity-domain violations in fact cause harm, whether to the self or to society at large. Furthermore, beliefs about self- and third-party harm predicted participants' moral attitudes toward impure acts.

\section{Study 2}


Study 1 revealed a correlation between harmfulness and immorality. As described in the introduction, this association could stem from reflection (i.e., harmfulness beliefs promoting moral condemnation) and/or rationalization (i.e., moral condemnation promoting harmfulness beliefs). Next, in Study 2, we simultaneously assess these hypothesized causal processes-by randomly assigning participants to either reflect or rationalize, and recording the outcome of each cognitive process.

Understanding the effects of these distinct cognitive processes may also offer insight into the nature of intergroup disagreements concerning the scope of the moral domain (Côté, Piff, \& Willer, 2013; Inbar et al., 2012; Hannikainen et al., 2017). In Study 1, for instance, lower SES theists were more likely to view impure acts as harmful and impermissible than were upper SES non-theists (see also Haidt et al., 1993; Piazza $\&$ Landy, 2013). Do these social groups differ in their moral values because they have contradictory beliefs about what is harmful? If so, the opportunity to focus on these divergent harm beliefs should aggravate their moral disagreement. Alternately, might disagreements about harmfulness emerge from differences in the groups' moral values? If so, the invitation to focus on their moral values should exacerbate disagreement about whether impure acts are harmful.

\subsection{Methods}

\subsubsection{Participants}

Students at a large, public university in Colombia were invited to take part in a study in exchange for course credit. 677 students $\left(221\right.$ women; $\left.M_{\text {age }}=22.6\right)$ followed through to completion. The distribution across socioeconomic strata was roughly representative of the Bogotá population (see Table 1).

Table 1. Distribution by social stratum, in comparison to 2014 census data.

\begin{tabular}{lcccccc}
\hline \multicolumn{7}{c}{ Social strata } \\
& $\mathbf{1}$ & $\mathbf{2}$ & $\mathbf{3}$ & $\mathbf{4}$ & $\mathbf{5}$ & $\mathbf{6}$ \\
\hline Study 1 & 17 & 60 & 78 & 18 & 7 & 3 \\
& $(9.3 \%)$ & $(32.8 \%)$ & $(42.6 \%)$ & $(9.8 \%)$ & $(3.8 \%)$ & $(1.6 \%)$ \\
Study 2 & 55 & 179 & 303 & 115 & 15 & 8 \\
& $(8.1 \%)$ & $(26.4 \%)$ & $(44.8 \%)$ & $(17.0 \%)$ & $(2.2 \%)$ & $(1.2 \%)$ \\
$\begin{array}{l}\text { Bogotá census data } \\
\text { (DANE, 2014) }\end{array}$ & $10.4 \%$ & $41.3 \%$ & $36.0 \%$ & $7.8 \%$ & $2.6 \%$ & $1.9 \%$ \\
\hline
\end{tabular}

\subsubsection{Procedure}

In a 2 (Focus: Reflection, Rationalization) $\times 2$ (Target: Self, Third-party) betweensubjects designs, participants were randomly assigned to one of four groups and asked to read three scenarios each describing an agent's conduct. We selected two scenarios from Study 1-Incest, and Chicken-and added a third scenario, Hoard—which we employ as a test of our manipulation: 
Richard, a wealthy entrepreneur, lives in a town in the mountains. During the winter months, the town faces food shortages affecting the local supermarkets. Some neighbors go hungry.

When food supplies are scarce, Richard buys more food at the supermarket than his family can possibly eat to make sure they are well fed.

As shown in Table 2, participants made a pre-judgment and then underwent a treatment requiring at least 20 seconds of reflection.

Table 2

Study 2: Experimental protocol.

\begin{tabular}{lll}
\hline \multicolumn{1}{c}{ Reflection condition } & \multicolumn{1}{c}{ Rationalization condition } \\
\hline \multirow{2}{*}{ Pre- judgment } & $\begin{array}{l}\text { Do you think that [Agent]'s behavior } \\
\text { should be allowed or forbidden? }\end{array}$ & $\begin{array}{l}\text { Do you think that [Agent]'s behavior } \\
\text { caused harm to [Target]? }\end{array}$ \\
& $\begin{array}{l}\text { Take a moment to think about } \\
\text { whether [Agent]'s behavior caused } \\
\text { Treatment }\end{array}$ & $\begin{array}{l}\text { Take a moment to think about } \\
\text { whether [Agent]'s behavior should be } \\
\text { allowed or forbidden, and why. }\end{array}$ \\
& $\begin{array}{l}\text { Do you think that [Agent]'s behavior } \\
\text { caused harm to [Target]? }\end{array}$ & $\begin{array}{l}\text { Do you think that [Agent]'s behavior } \\
\text { should be allowed or forbidden? }\end{array}$ \\
& $\begin{array}{l}\text { Now that you have thought about } \\
\text { whether [Agent]'s behavior caused } \\
\text { harm to [Target], we want to ask you } \\
\text { again about your beliefs about } \\
\text { whether [Agent]'s behavior should be } \\
\text { allowed or forbidden. }\end{array}$ & $\begin{array}{l}\text { Now that you have thought about } \\
\text { whether [Agent]'s behavior should be } \\
\text { allowed or forbidden, we want to ask } \\
\text { you again about whether [Agent]'s } \\
\text { behavior caused harm to [Target]. }\end{array}$ \\
& $\begin{array}{l}\text { You may or may not have changed } \\
\text { your mind. }\end{array}$ & $\begin{array}{l}\text { You may or may not have changed } \\
\text { your mind. }\end{array}$ \\
& $\begin{array}{l}\text { Do you think that [Agent]'s behavior } \\
\text { should be allowed or forbidden? }\end{array}$ & $\begin{array}{l}\text { Do you think that [Agent]'s behavior } \\
\text { caused harm to [Target]? }\end{array}$ \\
\hline
\end{tabular}

For instance, in the Reflection condition, participants first evaluated the action's permissibility after reading the scenario. On the following page, participants reflected on the harmfulness of the agent's conduct either for the agent herself or for "others, including her community and society", and were asked to free-write about their conclusions. After the manipulation, we asked participants to evaluate the action's permissibility a second time:

Now that you have thought about whether Richard's behavior caused harm to others, including his community and society at large, we want to ask you again about your beliefs about whether Richard's behavior should be allowed or forbidden.

You may or may not have changed your mind.

Do you think that Richard's behavior should be allowed or forbidden? 
Participants repeated this process for all three scenarios in a random order. In order to mitigate demand characteristics, we employed balanced language ("should be allowed or forbidden, and why"; "caused harm to [Target], and how or how not") that would not predispose either conclusion, or create the sense that either attitude change or stability is preferable ("You may or may not have changed your mind"; emphases added).

In the Rationalization condition, the order of the judgments was reversed-as shown in Table 2: Participants first assessed whether the behavior was harmful. They were then asked to ruminate on the moral status of the behavior in question and explain their moral judgment, and finally whether the behavior was harmful a second time.

Pre- and post-judgments were recorded on nine-point scales, and min-max normalized $(0=$ 'no harm' to $1=$ 'very much harm'; $0=$ 'definitely allowed' to $1=$ 'definitely forbidden'). We then calculated pre-to-post change scores (ranging from -1 to +1) and report them as percentages. Thus, the coefficients can be straightforwardly interpreted as the proportion (or percentage) of attitude change in terms of absolute change (e.g., from 'definitely allowed' to 'definitely forbidden', or vice versa). So, for instance, $+10 \Delta \%$ would indicate an increase of 0.8 on a nine-point scale.

Finally, participants provided information about their age, gender, administrative social stratum (from 1 to 6 ), and stated whether they believe in God (1: "Yes"; 0: "No").

\subsubsection{Pilot data}

To ascertain whether our experimental manipulation suffices to detect moralization via harm-based reflection, we first recruited 72 native English speakers $\left(M_{\text {age }}=34 ; 51 \%\right.$ women $)$ through Prolific.ac. As predicted, we observed increased moralization in the Hoard scenario after reflecting on third-party harm $\left(M_{\text {-post }}=58.9\right.$, $S D_{\text {post }}=32.6 ;$ vs. $M$-pre $\left.=55.8, S D_{\text {-pre }}=30.4\right), t(71)=2.43, p=.018, d_{\mathrm{z}}=0.29$.

\subsubsection{Analysis and predictions}

We pre-registered hypotheses and an analysis plan at aspredicted.org/zr8kd.pdf. Our analysis plan consisted of two analyses for each treatment: First, in an intent-totreat analysis (ITT), we evaluate the overall (positive or negative) effect of treatment assignment on attitude change using a linear-mixed effects model with random intercepts for each participant.

For instance, the ITT analysis in the Reflection condition evaluates whether reflecting on harm exerts a main effect (either positive or negative) on moral judgment (i.e., against the null hypothesis of no attitude change):

$$
\Delta_{i j k}=\mu+\alpha_{i}+\eta_{k(i)}+\beta_{j}+(\alpha \beta)_{i j}+\varepsilon k(i j)
$$

This mixed-effects model allows us to model attitude change for target $i$ and scenario $j$ for participant $k$ as a linear function of the overall mean $\mu$ plus the effects of Target $\alpha_{i}$, 
and Scenario $\beta_{j}$, the random effect of participant within target $\eta_{k(i)}$, the effect of scenario $\tau_{k}$, the effect of the interaction between target and scenario $(\alpha \beta)_{i j}$, and the error $\varepsilon_{k}(i j)$.

We are interested in whether the overall mean, intercept $\mu$, differs from zero (i.e., no change). ${ }^{2}$ The most straightforward test of the reflection model predicts that $\mu$ should be positive in the Reflection condition. This result would indicate that, as with prototypically harmful behavior (e.g., the Hoard vignette), focusing on the harmfulness of impure and indecent acts promotes their moralization.

A wide literature on reflectivity and moral judgment motivates the opposite directional prediction (Hannikainen et al., 2017; Paxton, Ungar \& Greene, 2012; Pennycook, Cheyne, Barr, Koehler, \& Fugelsang, 2014; Royzman, Landy \& Goodwin, 2014): i.e., that $\mu$ should be negative in the Reflection condition. Collectively, these studies demonstrate that reflective individuals are more likely to hold permissive attitudes toward the purity domain than are intuitive individuals-suggesting that the experimental induction of reflection should tend to promote more permissive attitudes.

Turning to the Rationalization condition, the most straightforward application of the rationalization hypothesis would predict that $a$ should be positive- such that a focus on the immorality of taboo acts elevates beliefs in their harmfulness.

However, assignment to a treatment condition does not guarantee compliance with, or susceptibility to, treatment. In our present case, participants who are asked to reflect on an act's harmfulness or immorality may not necessarily conclude that the act is harmful or immoral. So, we conducted additional treatment-on-the-treated analyses (TOT; see Geneletti \& Dawid, 2011) to evaluate the effect of reflection (or rationalization) among participants who conclude that the target acts are harmful (or immoral).

The TOT analysis differs from the ITT analysis due to the inclusion of an additional predictor, $\mathrm{\gamma} k(i j)$. For instance, in the TOT analysis of reflection, we define $\mathrm{\gamma} k(i j)$ as the harmfulness belief (reported during the manipulation) to target $i$ in scenario $k$ according to participant $j$ :

$$
\Delta_{i j k}=\mu+\alpha_{i}+\eta_{k(i)}+\beta_{j}+(\alpha \beta)_{i j}+\mathrm{\gamma} k(i j)+\varepsilon_{k(i j)}
$$

A positive $\gamma$ coefficient would provide qualitatively distinct evidence in support of the reflection model. ${ }^{3}$ While the ITT analysis asks whether reflection promotes condemnation overall, the TOT analysis asks whether reflection promotes condemnation

2 Our pre-registered analysis was a mixed model with two within-subjects factors (Scenario and Trial) and a between-subjects factor (Target), but we simplified the analysis by computing the difference score (for Trial), leaving us with one within- (Scenario) and one between- (Target) subjects factor. To render the model intercept interpretable as the overall attitude change (equivalent to the main effect of Trial) across scenarios and targets, we employ deviation coding (i.e., -0.5, 0.5) instead of dummy coding (i.e. 0, 1).

${ }^{3}$ As in footnote 1 , our analysis deviated from the pre-registered model in two ways: by employing a difference score approach, and deviation (instead of dummy) coding to render the intercept a main effect (rather than a simple effect). 
insofar as acts are viewed as harmful. (Similarly, a positive $\gamma$ in the Rationalization condition would indicate that opportunities to moralize promote harmfulness beliefs to the extent that acts are perceived as immoral.)

Notice, however, that only the IIT analysis preserves random assignment, thus providing the strongest test of a causal relation (Gupta, 2011). TOT analyses, by contrast, are potentially biased by a confounding variable that promotes both susceptibility to treatment and attitude change (Ten Have et al., 2008).

\subsection{Results}

Median reflection time in the Harm focus condition was $90.1\left[Q_{1}=55.2 ; Q_{3}=148.7\right]$ seconds, and median rumination time in the Morality focus condition was $80.7\left[Q_{1}=\right.$ $\left.37.9 ; Q_{3}=141.8\right]$ seconds — vastly exceeding the required twenty seconds.

\subsubsection{Effects of reflection}

As in our pilot United Kingdom study, reflection on third-party harm promoted opposition in the Hoarding case, $\mu=5.18 \Delta \%$, 95\% CI [3.19, 7.18], $t(175)=5.14, p<.001$, $d_{\mathrm{z}}=0.40,95 \%$ CI $[0.25,0.55]$. Having ascertained that the reflection induction drives opposition to unjust behavior, we now turn to the purity domain to assess whether comparable effects emerge when reflecting on the harmfulness of impure and indecent acts.

The ITT analysis of purity violations did not yield support for the reflection model: The effect of the reflection treatment was not significant, $\mu=0.10 \Delta \%, 95 \%$ CI [$1.12,1.49$ ], $t=0.14, p=.89$ (see break-down by scenario in Table 3 ).

However, the TOT analysis revealed that reported harm beliefs did predict change in moral judgment, $\gamma=13.53 \Delta \%, 95 \%$ CI $[9.31,17.76], t=6.30, p<.001$ : Specifically, participants who reported greater harmfulness tended to grow more opposed toward impure acts, while those reporting minimal harm tended to become more permissive (see Fig. 2).

\subsubsection{Effects of rationalization}

The ITT analysis yielded support for the rationalization model: Participants tended to view impure acts as more harmful after (versus before) ruminating on their moral status, $\mu=3.00 \Delta \%, 95 \%$ CI [1.71, 4.28], $t=4.58, p<.001$ (see Fig. 2 and Table $3) .4$

In contrast, the TOT analysis was not statistically significant: Differences in moral attitudes did not predict change in harm beliefs, $\gamma=3.24 \Delta \%, 95 \%$ CI $[-0.60,7.06]$, $t=1.66, p=.097$ - though the association trended in the predicted direction.

${ }^{4}$ In an exploratory analysis, we observed that the predicted main effect of moralization on harm beliefs was moderated by target: Change in harm beliefs was greater for third-party harm than for self-harm, $B=4.22 \Delta \%, 95 \%$ CI [1.66, 6.79], $t=3.23, p=.001$. 

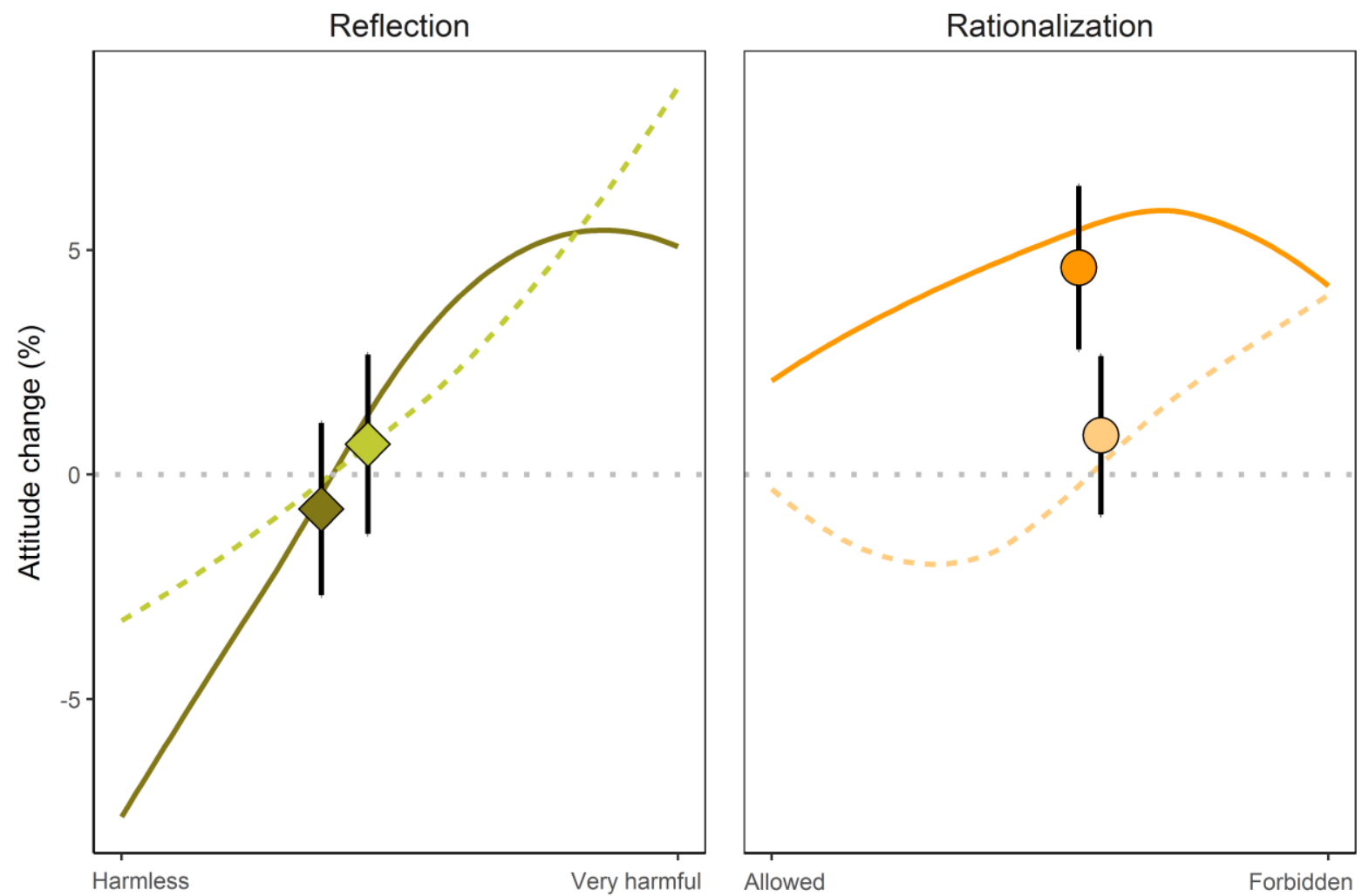

Figure 2. (Left) Change in moral judgment by third-party (solid line) and self- (dashed line) harm beliefs. (Right) Change in third-party (solid line) and self- (dashed line) harm beliefs by moral judgment. Regression lines are estimated with locally weighted smoothing. The point estimates (diamonds: Reflection; circles: Rationalization) display the ITT effects by target (lighter: self; darker: third-party) and their $95 \%$ confidence intervals.

Table 3. Study 2: Descriptive statistics and by-scenario t-tests.

\begin{tabular}{|c|c|c|c|c|c|c|c|c|c|}
\hline Condition & Target & Scenario & Pre & $I V$ & Post & $\Delta \%$ & $t$ & $p$ & Cohen's d \\
\hline \multirow{6}{*}{ Reflection } & \multirow{3}{*}{ Other } & Chicken & 51.3 & 31.5 & 49.6 & $\begin{array}{c}-1.70 \\
{[-4.28,0.87]}\end{array}$ & -1.31 & .193 & -0.10 \\
\hline & & Hoard & 52.8 & 59.9 & 58.0 & $\begin{array}{c}5.18 \\
{[3.19,7.18]}\end{array}$ & 5.14 & .000 & 0.39 \\
\hline & & Incest & 56.5 & 41.1 & 56.0 & $\begin{array}{c}-0.57 \\
{[-3.24,2.11]}\end{array}$ & -0.42 & .675 & -0.03 \\
\hline & \multirow{3}{*}{ Self } & Chicken & 56.8 & 43.0 & 58.7 & $\begin{array}{c}2.29 \\
{[-0.44,5.03]}\end{array}$ & 1.65 & .100 & 0.13 \\
\hline & & Hoard & 51.9 & 20.3 & 51.7 & $\begin{array}{c}-0.15 \\
{[-2.38,2.09]}\end{array}$ & -0.13 & .897 & -0.01 \\
\hline & & Incest & 61.8 & 46.3 & 61.8 & $\begin{array}{c}0.37 \\
{[-2.68,3.42]}\end{array}$ & 0.24 & .811 & 0.02 \\
\hline \multirow{2}{*}{ Rationalization } & \multirow{2}{*}{ Other } & Chicken & 31.4 & 52.9 & 36.4 & $\begin{array}{c}4.95 \\
{[2.13,7.78]}\end{array}$ & 3.47 & .001 & 0.27 \\
\hline & & Hoard & 55.7 & 53.3 & 57.1 & $\begin{array}{c}1.14 \\
{[-0.94,3.22]}\end{array}$ & 1.09 & .279 & 0.08 \\
\hline
\end{tabular}




\begin{tabular}{ccccccccc} 
& Incest & 35.2 & 55.6 & 40.5 & $\begin{array}{c}5.26 \\
{[2.43,8.08]}\end{array}$ & 3.68 & .000 & 0.29 \\
\hline & Chicken & 57.9 & 60.6 & 58.4 & $\begin{array}{c}0.74 \\
{[-1.23,2.71]}\end{array}$ & 0.74 & .460 & 0.06 \\
Self & Hoard & 24.0 & 52.7 & 27.8 & $\begin{array}{c}3.62 \\
{[0.90,6.34]}\end{array}$ & 2.63 & .009 & 0.20 \\
& & & & & & \\
& Incest & 55.6 & 56.8 & 56.4 & $\begin{array}{c}1.03 \\
{[-1.36,3.42]}\end{array}$ & 0.85 & .396 & 0.07 \\
\hline
\end{tabular}

\subsubsection{Exploratory analyses of intergroup disagreement}

If intergroup moral disagreement arises from disagreements about harmfulness, consequentialist reflection should reinforce condemnation among low SES theists, and demoralization among high SES non-theists. The opportunity to focus on their contradictory beliefs should, on this view, aggravate moral disagreement between social groups.

Yet, we observed the opposite relation: The effect of harm reflection was associated positively to social strata, $\beta_{\mathrm{SES}}=1.86,95 \% \mathrm{CI}[0.43,3.29], t=2.56, p=.010$. In other words, low SES participants tended to become more lenient toward impure acts while high SES participants became slightly more opposed. A similar effect was observed when comparing theists to non-theists, $\beta_{\text {Theism }}=-3.10 \Delta \%, 95 \%$ CI $[-5.90,-0.29]$, $t=2.17, p=.031$ : The reflection manipulation tended to mitigate moral disagreement between theists and non-theists-though neither simple effect was statistically significant (theists: $\mu=-1.72 \Delta \%, t=-1.81, p=.071$; non-theists: $\mu=1.37 \Delta \%, z=1.35, p$ $=.18$ ). Thus, the overall pattern of results provided evidence against the prediction that intergroup moral disagreements arise from processes of consequentialist reflection.

We also examined whether intergroup differences in harmfulness beliefs arise from moral disagreement. If so, we should expect the effect of rationalization to be larger among low SES than among high SES participants. The opportunity to focus on their divergent moral values should, on this view aggravate disagreement between social groups about whether harm ensued.

Indeed, beliefs about harmfulness increased among participants in lower, but not upper, social strata via rationalization (see Fig. 3) - though the linear effect was not significant, $\beta_{\mathrm{SES}}=-1.21,95 \%$ CI [-2.56, 0.13], $t=-1.68, p=.078$. A corresponding pattern of results emerged when treating theism as a moderator, $\beta_{\text {Theism }}=3.06,95 \%$ CI $[0.49$, 5.62], $t=2.33, p=.020$ : Ruminating on the moral status of impure acts significantly elevated harmfulness beliefs among religious participants $(n=281), \mu=4.73 \Delta \%, t=$ $4.49, p<.001$, but not among the non-religious $(n=364), \mu=1.59 \Delta \%, t=1.63, p=.10$. 
Reflection $\bigcirc$ Rationalization
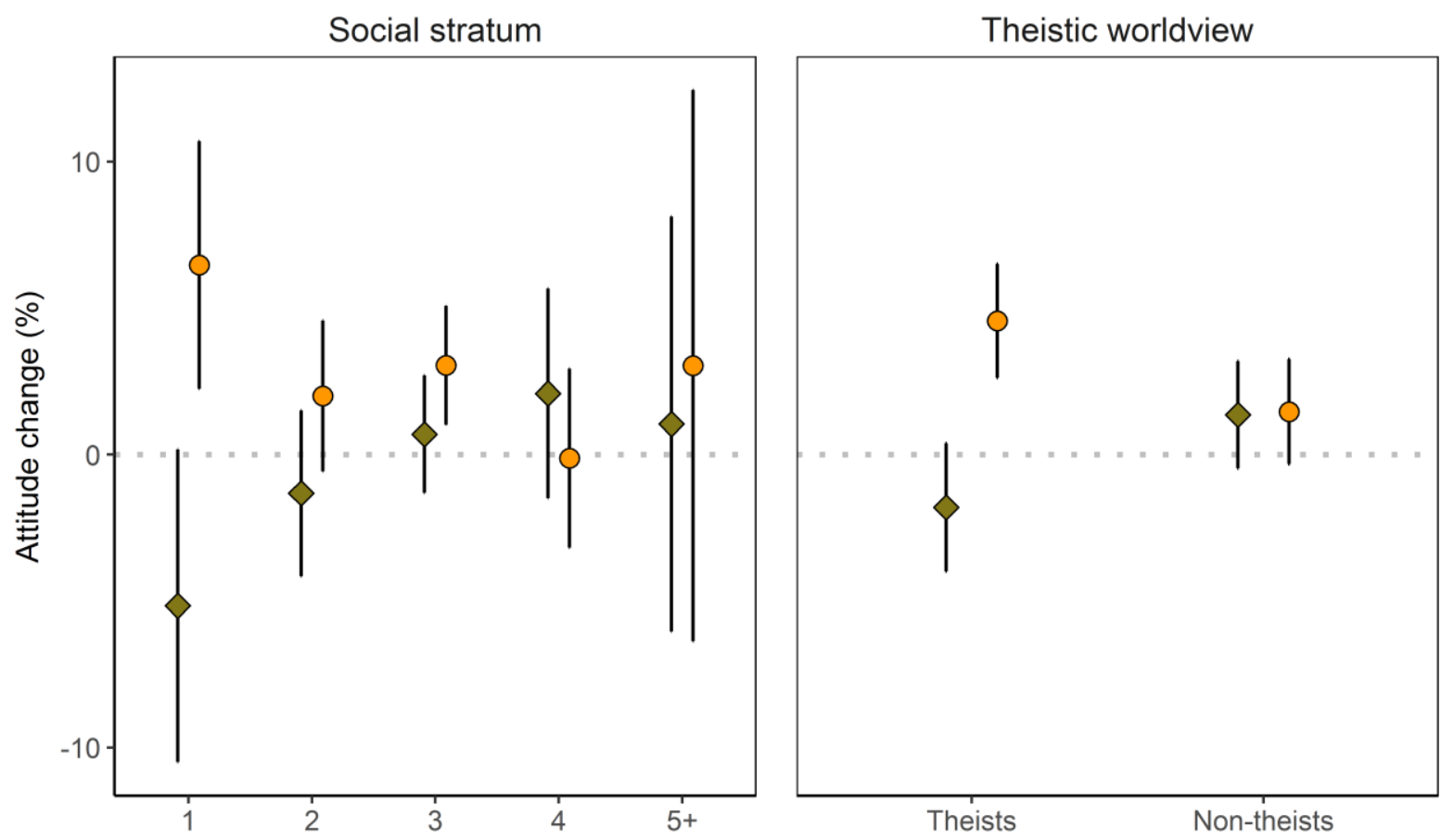

Figure 3. Attitude change in the Reflection (diamonds) and Rationalization (circles) conditions by social stratum (left) and worldview (right).

\subsection{Discussion}

In the harm domain, consequentialist reflection played a clear role inspiring moral condemnation. Initially, participants both in Colombia and the United Kingdom were divided regarding the moral status of hoarding, but they grew more opposed after a moment's reflection on whether it is harmful to others.

Meanwhile, in the purity domain, ruminating on an action's moral status tended to promote the belief that the act in question is harmful, particularly to third-parties. The magnitude of the increase, however, did not depend upon participants' moral attitudes (as shown by the TOT analysis). In contrast, we observed no overall change after harm reflection. Rather, the direction of moral attitude change depended on whether participants concluded upon reflection that harm ensued. Some reasoned that impure actions are harmful and then became more opposed; others concluded that they are harmless and tended to become more lenient. Together these effects resulted in no mean change.

Then, exploratory analyses suggested that rationalization-but not reflectionmay account for the emergence of intergroup disagreement: Low SES theists revealed a larger effect of rationalization than did upper SES non-theists; and, as a result, factual disagreement increased following moral rumination. In contrast, consequentialist reflection appeared to mitigate moral disagreement between social groups-plausibly as participants were generating new and counterintuitive reasons to oppose their initial moral judgments. 


\section{Study 3}

In Study 3, we investigate whether the effects of rationalization and reflection documented among students in Colombia are also observed in the United Kingdom. Our exploratory analyses of intergroup disagreement provided suggestive evidence that lower SES theists are more susceptible to these effects-and, in particular, to rationalization. The second objective of Study 3 is to understand why and how this occurs.

One possibility is that religious credence inherently promotes rationalization: For instance, some research shows that concern for purity and decency among religious individuals is fully mediated by the belief that morality is a set of divine commands (Piazza \& Landy, 2013). This core motive may also magnify harmfulness beliefs via rationalization.

Alternately, variation in susceptibility to reflection and rationalization across social groups may be rooted in a third variable. We hypothesized that this variable could be cognitive style (Frederick, 2005), for three related reasons: (1) Religious individuals typically adopt a more intuitive style of thought than do the non-religious (Shenhav, Rand \& Greene, 2012), and (2) the reliance on intuition is independently linked to stronger opposition to impure acts (Garvey \& Ford, 2014; Landy, 2016; Pennycook et al., 2014; Royzman et al., 2014), plausibly as (3) intuitive thinkers gravitate towards nonconsequentialist approaches to moral evaluation (Cushman, 2013; Hannikainen et al., 2017; Piazza \& Sousa, 2014).

Thus, in Study 3, we examine whether the outcomes of rationalization and reflection depend upon one's socioeconomic status and theistic worldview, and/or trait differences in domain-general reflectivity.

\subsection{Methods}

\subsubsection{Participants}

In order to ensure a uniform distribution across social strata and increase power to replicate the effect of socioeconomic status on rationalization and reflection, we employed an extreme-groups approach to stratified sampling.

Native English-speaking adults were recruited from the Prolific.ac panel and stratified based on enrolment data on socioeconomic status (in the form of a ten-rung ladder): 1-3 ("lower"), 5 ("middle"), and 8-10 ("upper"). We invited 145 participants (total $N=457 ; 250$ women; $M_{\text {age }}=35.8$ ) in each stratum-lower, middle, and upper-to take part in our experiment.

\subsubsection{Procedure}

In a between-subjects design, participants were randomly assigned to either the Reflection or Rationalization condition (as in Study 2), and viewed a subset of four scenarios describing violations of purity and decency norms drawn randomly from a set of eight (used in Hannikainen et al., 2017).

For each scenario, participants followed the same protocol used in Study 2 with two modifications: (1) They were asked how "morally wrong" each action is on a sevenpoint scale from "not wrong at all" to "very morally wrong". (2) Every participant was 
asked to reflect on harm "to others, including family, community and society at large". Thus, in Study 3 we dropped the previous 'self-harm' condition.

After evaluating four behaviors in sequence, participants completed the threeitem Cognitive Reflection Test (CRT; Frederick, 2005). We coded the number of intuitive responses (Cronbach's $a=.72$ ) as a measure of cognitive style.

Finally, participants answered two demographic questions, on religiosity ("Do you consider yourself a religious person?") and educational attainment ("What is the highest degree you have earned?").

\subsubsection{Analysis and predictions}

For each condition, we entered difference scores as the dependent measure in a mixed-effects linear regression with crossed random effects of participant and scenario, reporting the model intercept as the estimate of overall ITT effects.

Next, drawing on data from both conditions, we entered condition as a fixed effect to assess whether the ITT effects of rationalization and reflection statistically differ. Building on this model, we then entered a series of hypothesized moderators in separate analyses: (1) SES, (2) religiosity, and (3) CRT score. For each predictor, we report the Condition $\times$ Moderator interaction to understand individual differences in susceptibility to rationalization and reflection.

\subsection{Results}

Median reflection time in the Reflection condition was $45.5\left[Q_{1}=30.9 ; Q_{3}=70.9\right]$ seconds, and median rumination time in the Rationalization condition was $46.7\left[Q_{1}=\right.$ 33.3; $\left.Q_{3}=71.2\right]$ seconds.

In the Reflection condition, participants tended to demoralize impure acts, $\mu=$ $4.89 \Delta \%, 95 \%$ CI [-7.55, -2.22], $t=-3.81, p<.001$-in line with prior work on cognitive reflection and consequentialist moral judgment (Hannikainen et al., 2017; Paxton et al., 2012; Pennycook et al., 2014; Royzman et al., 2014). The corresponding TOT analysis was also statistically significant, $\gamma=9.18 \Delta \%, 95 \%$ CI $[4.73,13.55], t=4.13, p<.001$.

Replicating Study 2, the opportunity to morally evaluate impure acts in the Rationalization condition promoted beliefs in their harmfulness, $\mu=1.92 \Delta \%, 95 \% \mathrm{CI}$ $[0.74,3.11], t=3.32, p<.001$. This time the TOT effect was statistically significant, $\gamma=$ $6.43 \Delta \%, 95 \%$ CI $[3.88,8.93], t=5.03, p<.001$.

The difference between the ITT effects of reflection versus rationalization was highly significant, $t=8.29, p<.001$, and emerged separately by scenario for seven out of eight scenarios (see Table 4).

Table 4. Study 3: Descriptive statistics and by-scenario t-tests.

\begin{tabular}{|c|c|c|c|c|c|c|c|c|}
\hline Condition & Scenario & Pre- & $I V$ & Post- & $\Delta \%[95 \% C I]$ & $t$ & $p$ & Cohen's d \\
\hline \multirow{3}{*}{ Reflection } & Artist & 15.6 & 9.6 & 14.1 & $\begin{array}{c}-1.52 \\
{[-3.72,0.68]}\end{array}$ & -1.37 & .175 & -0.13 \\
\hline & Chicken & 65.9 & 28.3 & 55.7 & $\begin{array}{c}-10.20 \\
{[-14.53,-5.87]}\end{array}$ & -4.67 & .000 & -0.43 \\
\hline & French-kiss & 45.1 & 35.1 & 41.8 & $\begin{array}{c}-3.33 \\
{[-6.24,-0.43]}\end{array}$ & -2.27 & .025 & -0.21 \\
\hline
\end{tabular}




\begin{tabular}{|c|c|c|c|c|c|c|c|c|}
\hline & Hair & 36.9 & 9.5 & 29.3 & $\begin{array}{c}-7.93 \\
{[-11.32,-4.54]}\end{array}$ & -4.63 & .000 & -0.43 \\
\hline & Smear & 44.3 & 17.7 & 36.5 & $\begin{array}{c}-7.85 \\
{[-11.79,-3.91]}\end{array}$ & -3.95 & .000 & -0.37 \\
\hline & Soul & 22.6 & 20.9 & 23.1 & $\begin{array}{c}0.68 \\
{[-2.14,3.50]}\end{array}$ & 0.48 & .634 & 0.04 \\
\hline & Tail & 25.8 & 17.6 & 20.2 & $\begin{array}{c}-4.96 \\
{[-8.78,-1.13]}\end{array}$ & -2.57 & .012 & -0.24 \\
\hline & Urinate & 53.2 & 42.2 & 49.3 & $\begin{array}{c}-3.91 \\
{[-7.96,0.14]}\end{array}$ & -1.91 & .058 & -0.18 \\
\hline \multirow{8}{*}{ Rationalization } & Artist & 6.9 & 16.5 & 9.9 & $\begin{array}{c}3.38 \\
{[1.26,5.49]}\end{array}$ & 3.16 & .002 & 0.29 \\
\hline & Chicken & 23.4 & 54.9 & 26.9 & $\begin{array}{c}3.48 \\
{[0.24,6.71]}\end{array}$ & 2.13 & .035 & 0.20 \\
\hline & French-kiss & 21.9 & 38.9 & 25.4 & $\begin{array}{c}3.33 \\
{[0.69,5.98]}\end{array}$ & 2.49 & .014 & 0.23 \\
\hline & Hair & 13.8 & 32.8 & 13.4 & $\begin{array}{c}-0.34 \\
{[-1.67,0.99]}\end{array}$ & -0.50 & .615 & -0.05 \\
\hline & Smear & 16.5 & 37.2 & 18.4 & $\begin{array}{c}1.95 \\
{[-0.99,4.90]}\end{array}$ & 1.31 & .191 & 0.12 \\
\hline & Soul & 19.0 & 21.4 & 20.0 & $\begin{array}{c}1.03 \\
{[-1.11,3.16]}\end{array}$ & 0.95 & .342 & 0.09 \\
\hline & Tail & 17.7 & 22.6 & 17.9 & $\begin{array}{c}0.46 \\
{[-1.45,2.36]}\end{array}$ & 0.47 & .637 & 0.04 \\
\hline & Urinate & 42.6 & 53.4 & 44.8 & $\begin{array}{c}2.14 \\
{[-0.36,4.64]}\end{array}$ & 1.70 & .093 & 0.16 \\
\hline
\end{tabular}

\subsubsection{Socioeconomic status}

Although we achieved very good balance in socioeconomic status (Bottom $5=$ " $2^{\text {nd }}$ rung", $Q_{1}=$ " $3^{\text {rd }}$ rung", $M d n=$ " $5^{\text {th }}$ rung", $Q_{3}=$ " $8^{\text {th }}$ rung", Top $95=$ " $9^{\text {th }}$ rung"), the effect of condition was no smaller among upper SES participants. Namely, the two-way interaction between SES and condition was not significant, $\beta=0.24 \Delta \%, 95 \%$ CI [-0.47, $0.94], t=0.66, p=.51$.

\subsubsection{Theistic worldview}

While our sample in Study 3 was largely non-religious, theists $(n=95)$ were no more susceptible to the effects of rationalization and reflection than were non-theists $(n$ $=341$ ) - according to the corresponding two-way interaction, $\beta=2.68 \Delta \%, 95 \%$ CI $[-1.12$, 6.53], $t=1.36, p=.17$.

Thus, in contrast with the re-analyses of Study 2, sociodemographic characteristics in Study 3 failed to predict participants' susceptibility to rationalization and reflection.

\subsubsection{Cognitive style}


In a multiple regression, CRT scores were associated with SES, $\beta=-0.08,95 \%$ CI [-0.13, -0.03], $t=-3.16, p=.002$, but not religiosity, $\beta=0.09,95 \%$ CI $[-0.17,0.35], t=$ $0.67, p=.51 .5$

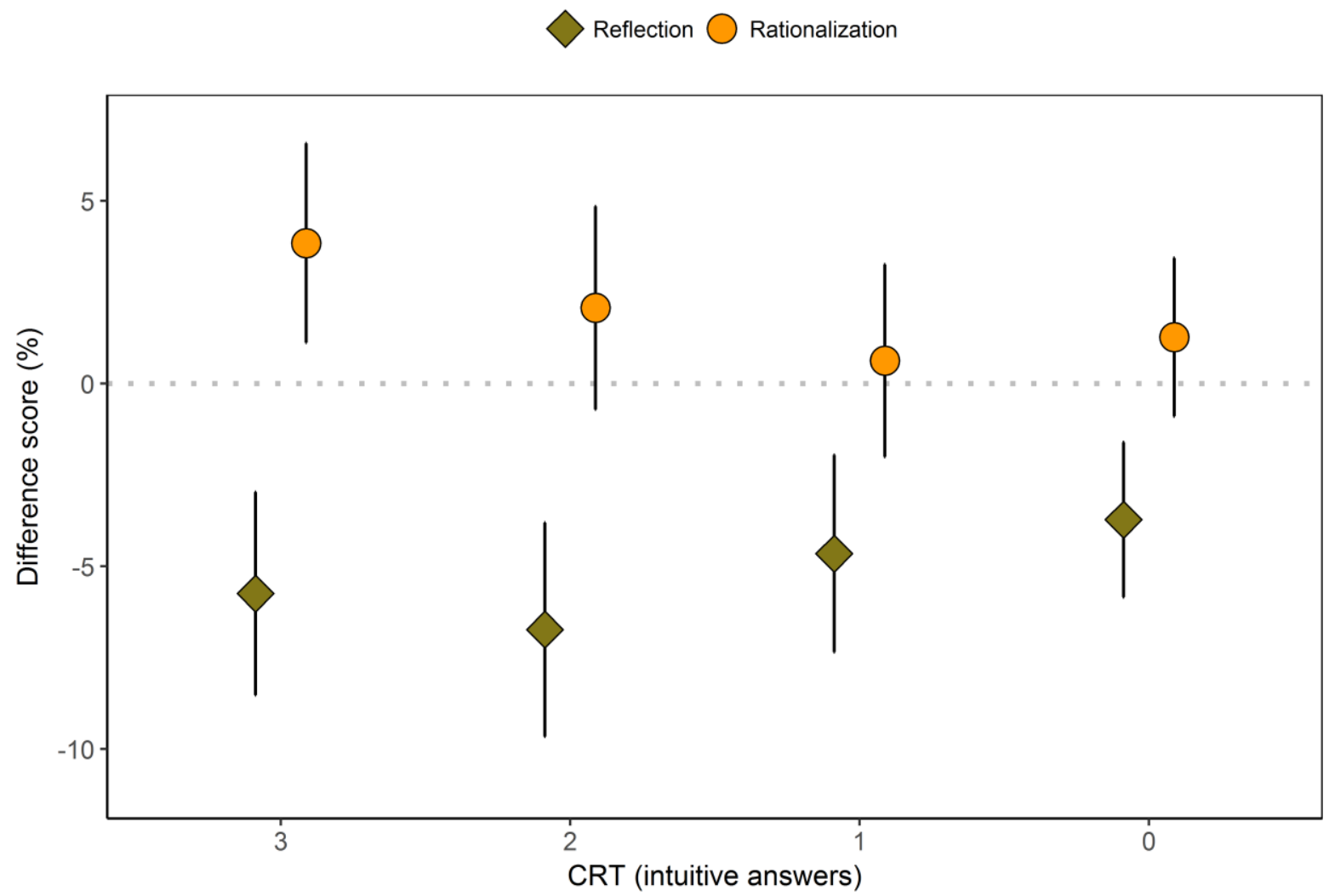

Figure 4. Attitude change due to reflection and rationalization by CRT score.

Entering condition, CRT score, and the condition $\times$ CRT score interaction revealed the predicted two-way interaction: $\beta=0.71 \Delta \%, 95 \%$ CI [0.08, 2.85], $t=2.07, p=.039 .{ }^{6} \mathrm{As}$ shown in Fig. 4, susceptibility to the effects of rationalization and reflection was greatest among intuitive thinkers.

\subsection{Discussion}

In Study 3, we examined the consequences of rationalization and reflection in a sample of United Kingdom residents. We replicated the positive effect of rationalization, and observed a negative effect of reflection - that did not emerge among Colombians.

5 This analysis also replicated the effect of familiarity (Stieger \& Reips, 2016): Using number of complete sessions as a proxy for non-naiveté, we also found that experienced study participants reported fewer intuitive answers than did beginners, $\beta=-0.09,95 \%$ CI [-0.15, -0.04], $t=-3.24, p$ $<.001$.

6 Some work has shown that CRT scores increase with familiarity (Stieger \& Reips, 2016), and thereby lose predictive validity. In an exploratory analysis, we entered participant expertise (the number of approved Prolific.ac submissions) as a proxy for familiarity. Indeed, the predicted two-way interaction was notably stronger in this analysis: $\beta=1.82 \Delta \%, 95 \% \mathrm{CI}[0.40$, $3.24], t=2.50, p=.013$. 
We also found that cognitive style moderated the divergent effects of rationalization and reflection. Meanwhile, demographic differences linked to socioeconomic status and religiosity did not generalize to the UK sample-perhaps because greater inequality drives more dramatic cultural differences in Colombia than in the United Kingdom.

Reflective thinkers revealed a weaker effect of the manipulations: Possibly, their pre-induction judgments adhere to consequentialist principles to a greater extent (Cushman, 2013; Greene, 2008), thus attenuating the effect that momentary reflection has on their reassessments.

\section{General discussion}

Sexual and culinary taboos have long been hailed as evidence that the moral domain is diverse (Chakroff, Dungan \& Young, 2013; Shweder et al., 1997; Graham, Haidt \& Nosek, 2009), and that moralization does not always depend on representations involving harm (Nussbaum, 2010; Rozin, Haidt \& Fincher, 2009; Tybur, Lieberman, \& Griskevicius, 2009). Yet, other research has shown that moral disapproval in these domains is best predicted by beliefs about harmfulness (Schein et al., 2016), and not disgust (Gray \& Schein, 2016).

In Study 1, we replicated this result: Moral disapproval of impure acts was accompanied by the belief that they incur harm to the self and to society at large. Then, in subsequent experiments (Studies 2 and 3) we recreated two cognitive processes that could account for the observed relationship between harm and immorality: one, in which reflecting on harmfulness promotes moralization; another, in which moralization promotes the belief in harmfulness.

We randomly assigned Colombian and British participants to engage in either of these processes-reflection or rationalization-and recorded the effects of each manipulation on their values and beliefs. Did participants' grow more opposed to taboo acts after focusing on their harmfulness, or perceive them as more harmful after focusing on their moral status?

The former effect of consequentialist reflection varied across cultures and domains: While consequentialist reflection promoted moralization in the harm domain in both cultures, effects in the purity domain differed-negative in the United Kingdom, but not in Colombia.

Meanwhile, rationalization in the purity domain was ubiquitous: Focusing on the moral status of impure and indecent acts elevated beliefs in their harmfulness in both cultures (Gray et al., 2014). Furthermore, these effects appeared more pronounced among religious and low-SES participants in Colombia; though Study 3 provided evidence that these associations likely stem from corresponding differences in cognitive style (Shenhav et al., 2012). Thus, our studies help to advance our understanding of the relationship between harm and morality (Gray et al., 2014; Haidt, 2001; Sousa, Holbrook \& Piazza, 2009; Turiel, 1983), by demonstrating divergent effects of two cognitive processes connecting these related constructs and elucidating their impact on intergroup disagreement.

One may wonder, though, whether our results instead reflect demand characteristics of the general experimental protocol. Although we cannot rule out some 
degree of acquiescence in general, the protocol was identical across conditions and only the sequence of judgments was allowed to differ. Thus, the divergence between the experimental effects of rationalization versus reflection cannot be the result of demand characteristics alone.

Though we documented rationalization effects in the moral domain, our studies generated limited insight regarding the mechanism by which moral evaluation enhances the perception of harm. One possibility is that a focus on an act's morality directs attention away from its outcomes (Miller, Hannikainen \& Cushman, 2013) and toward independent grounds for condemnation (Everett, Pizarro \& Crockett, 2016; Tepe \& Aydinli-Karakulak, 2019; Uhlmann, Pizarro, \& Diermeier, 2015). If so, after a moral assessment, when asked to rate the action's harmfulness again, participants may be embracing harmfulness as a reasonable explanation for their own moral opposition (Cushman, 2019) - concluding that the act is in fact not as innocuous as they had previously thought.

We must also note that, in practice, most moral evaluation is unlikely to involve discrete episodes of either rationalization or reflection-but probably implicates both processes to varying degrees, and reflects their mutual effects upon each other (Gray, Schein \& Cameron, 2017).

Our emphasis has been on the robustness of rationalization effects, but variation in reflection effects was also quite dramatic. Previous literature demonstrates that trait reflectivity is linked to demoralization of purity-related issues (Paxton et al., 2012; Pennycook et al., 2014; Royzman et al., 2014): i.e., reflective individuals tend to view impure acts as harmless and therefore permissible on consequentialist grounds. In our present study, we observed convergent experimental evidence for this effect only in the British sample, which suggests a potential limit on its generalizability. In the more collectivist and religious culture (i.e., Colombia), consequentialist reflection had no overall effect (see also McPhetres, Conway, Hughes, \& Zuckerman, 2018).

Insofar as our methods offer a window into moralization in the real world (see Inbar et al., 2009), we draw two closing insights. First, disputes about the ethics of abortion or minority rights, for instance, are presumed to emerge from broader worldview differences-yet our present evidence suggests that they also help to crystallize them. In other words, divergent moral values may in fact perpetuate disagreements about factual matters (Liu \& Ditto, 2013), such as the consequences of different policies surrounding culture war issues. Second, and more promisingly, we found grounds to hope that unguided consequentialist reflection could alleviate some of this moral discord. 


\section{References}

Blair, R. J. R. (1995). A cognitive developmental approach to morality: Investigating the psychopath. Cognition, 57(1), 1-29. https://doi.org/10.1016/0010-0277(95)00676-p

Côté, S., Piff, P. K., \& Willer, R. (2013). For whom do the ends justify the means? Social class and utilitarian moral judgment. Journal of Personality and Social Psychology, 104(3), 490. https://doi.org/10.1037/a0030931

Cushman, F. (2013). Action, outcome, and value: A dual-system framework for morality. Personality and Social Psychology Review, 17(3), 273-292. https://doi.org/10.1177/1088868313495594

Cushman, F. (2019). Rationalization is rational, manuscript in preparation.

Chakroff, A., Dungan, J., \& Young, L. (2013). Harming ourselves and defiling others: What determines a moral domain?. PloS one, 8(9), e74434. https://doi.org/10.1371/journal.pone.0074434

DANE -- Departamento Administrativo Nacional de Estadística. (2014). Encuesta multipropósito 2014. Available at: https://www.dane.gov.co/files/investigaciones/multi/Anexos_EM_2014.xls

Ditto, P. H., Pizarro, D. A., \& Tannenbaum, D. (2009). Motivated moral reasoning. Psychology of learning and motivation, 50, 307-338. https://doi.org/10.1016/s0079-7421(08)00410-6

Everett, J. A., Pizarro, D. A., \& Crockett, M. J. (2016). Inference of trustworthiness from intuitive moral judgments. Journal of Experimental Psychology: General, 145(6), 772. https://doi.org/10.1037/xge0000165

Frederick, S. (2005). Cognitive reflection and decision making. Journal of Economic Perspectives, 19(4), 25-42. https://doi.org/10.1257/089533005775196732

Garvey, K. J., \& Ford, T. G. (2014). Rationality, political orientation, and the individualizing and binding moral foundations. Letters on Evolutionary Behavioral Science, 5(1), 9-12. https://doi.org/10.5178/lebs.2014.29

Geneletti, S., \& Dawid, A. P. (2011). Defining and identifying the effect of treatment on the treated. In Illari, P. M., Russo, F., \& Williamson, J. (Eds.). Causality in the Sciences. Oxford University Press. https://doi.org/10.1093/acprof:oso/9780199574131.003.0034

Graham, J., Haidt, J., \& Nosek, B. A. (2009). Liberals and conservatives rely on different sets of moral foundations. Journal of personality and social psychology, 96(5), 1029. https://doi.org/10.1037/a0015141

Gray, K., \& Schein, C. (2016). No absolutism here: Harm predicts moral judgment 30× better than disgust-Commentary on Scott, Inbar, \& Rozin (2016). Perspectives on Psychological Science, 11(3), 325-329. https://doi.org/10.1177/1745691616635598

Gray, K., Schein, C., \& Cameron, C. D. (2017). How to think about emotion and morality: circles, not arrows. Current opinion in psychology, 17, 41-46. https://doi.org/10.1016/j.copsyc.2017.06.011

Gray, K., Schein, C., \& Ward, A. F. (2014). The myth of harmless wrongs in moral cognition: Automatic dyadic completion from sin to suffering. Journal of Experimental Psychology: General, 143(4), 1600. https://doi.org/10.1037/a0036149

Greene, J. D. (2008). The secret joke of Kant's soul. Moral psychology, 3, 35-79. https://doi.org/10.7551/mitpress/7504.003.0004

Gupta, S. K. (2011). Intention-to-treat concept: a review. Perspectives in clinical research, 2(3), 109. https://doi.org/10.4103/2229-3485.83221

Haidt, J. (2001). The emotional dog and its rational tail: a social intuitionist approach to moral judgment. Psychological review, 108(4), 814. https://doi.org/10.1037//0033-295x.108.4.814

Haidt, J. (2007). The new synthesis in moral psychology. Science, 316(5827), 998-1002. https://doi.org/10.1126/science.1137651 
Haidt, J., \& Hersh, M. A. (2001). Sexual morality: The cultures and emotions of conservatives and liberals. Journal of Applied Social Psychology, 31(1), 191-221. https://doi.org/10.1111/j.1559-1816.2001.tb02489.x

Haidt, J., Koller, S. H., \& Dias, M. G. (1993). Affect, culture, and morality, or is it wrong to eat your dog? Journal of Personality and Social Psychology, 65, 613- 628. https://doi.org/10.1037//0022-3514.65.4.613

Hannikainen, I. R., Miller, R. M., \& Cushman, F. A. (2017). Act versus impact: Conservatives and liberals exhibit different structural emphases in moral judgment. Ratio, 30(4), 462-493. https://doi.org/10.1111/rati.12162

Henrich, J., Heine, S., \& Norenzayan, A. (2010). The weirdest people in the world? Behavioral and Brain Sciences, 33(2-3), 61-83. https://doi.org/10.1017/s0140525x0999152x

Hofstede, G., Hofstede, G. J., \& Minkov, M. (2010). Cultures and organizations: Software of the mind.

Inbar, Y., Pizarro, D. A., \& Bloom, P. (2009). Conservatives are more easily disgusted than liberals. Cognition and Emotion, 23(4), 714-725. https://doi.org/10.1080/02699930802110007

Inbar, Y., Pizarro, D., Iyer, R., \& Haidt, J. (2012). Disgust sensitivity, political conservatism, and voting. Social Psychological and Personality Science, 3(5), 537-544. https://doi.org/10.1177/1948550611429024

Landy, J. F. (2016). Representations of moral violations: Category members and associated features. Judgment and Decision Making, 11, 496-508.

Lieberman, D., \& Lobel, T. (2012). Kinship on the Kibbutz: Coresidence duration predicts altruism, personal sexual aversions and moral attitudes among communally reared peers. Evolution and Human Behavior, 33(1), 26-34. https://doi.org/10.1016/j.evolhumbehav.2011.05.002

Liu, B. S., \& Ditto, P. H. (2013). What dilemma? Moral evaluation shapes factual belief. Social Psychological and Personality Science, 4(3), 316-323. https://doi.org/10.1177/1948550612456045

McPhetres, J., Conway, P., Hughes, J. S., \& Zuckerman, M. (2018). Reflecting on God's will: Reflective processing contributes to religious peoples' deontological dilemma responses. Journal of Experimental Social Psychology, 79, 301-314. https://doi.org/10.1016/j.jesp.2018.08.013

Mercier, H., \& Sperber, D. (2011). Why do humans reason? Arguments for an argumentative theory. Behavioral and brain sciences, 34(2), 57-74. https://doi.org/10.1017/s0140525x10000968

Miller, R. M., Hannikainen, I. R., \& Cushman, F. A. (2014). Bad actions or bad outcomes? Differentiating affective contributions to the moral condemnation of harm. Emotion, 14(3), 573. https://doi.org/10.1037/e505052014-080

Nichols, S. (2004). Sentimental rules: On the natural foundations of moral judgment. Oxford University Press. https://doi.org/10.1093/0195169344.001.0001

Nussbaum, M. C. (2010). From disgust to humanity: Sexual orientation and constitutional law. New York, NY: Oxford University Press.

Paxton, J. M., Ungar, L., \& Greene, J. D. (2012). Reflection and reasoning in moral judgment. Cognitive Science, 36(1), 163-177. https://doi.org/10.1111/j.1551-6709.2011.01210.x

Pennycook, G., Cheyne, J. A., Barr, N., Koehler, D. J., \& Fugelsang, J. A. (2014). The role of analytic thinking in moral judgements and values. Thinking \& Reasoning, 20(2), 188-214. https://doi.org/10.1080/13546783.2013.865000

Piazza, J., \& Landy, J. (2013). "Lean not on your own understanding": Belief that morality is founded on divine authority and non-utilitarian moral thinking. Judgment and Decision Making, 8(6), 639-661. 
Piazza, J., \& Sousa, P. (2014). Religiosity, political orientation, and consequentialist moral thinking. Social Psychological and Personality Science, 5(3), 334-342. https://doi.org/10.1177/1948550613492826

Royzman, E. B., Kim, K., \& Leeman, R. F. (2015). The curious tale of Julie and Mark: Unraveling the moral dumbfounding effect. Judgment and Decision Making, 10(4), 296-314.

Royzman, E. B., Landy, J. F., \& Goodwin, G. P. (2014). Are good reasoners more incest-friendly? Trait cognitive reflection predicts selective moralization in a sample of American adults. Judgment and Decision Making, 9(3), 176-190.

Rozin, P., Haidt, J., \& Fincher, K. (2009). From oral to moral. Science, 323(5918), 1179-1180. https://doi.org/10.1126/science.1170492

Schein, C., \& Gray, K. (2015). The unifying moral dyad: Liberals and conservatives share the same harm-based moral template. Personality and Social Psychology Bulletin, 41, 1147-1163. https://doi.org/10.1177/0146167215591501

Schein, C., Ritter, R. S., \& Gray, K. (2016). Harm mediates the disgust-immorality link. Emotion 16(6): 862-876. https://doi.org/10.1037/emo0000167

Shenhav, A., Rand, D. G., \& Greene, J. D. (2012). Divine intuition: Cognitive style influences belief in God. Journal of Experimental Psychology: General, 141(3), 423. https://doi.org/10.1037/e519702015-023

Shweder, R., Much, N., Mahapatra, M., \& Park, L. (1997). Divinity and the "big three" explanations of suffering. Morality and health, 119, 119-169.

Sivananda, S. (1934). Practice of brahmacharya. Yoga Vedanta Forest University, Divine Life Society.

Sousa, P., Holbrook, C., \& Piazza, J. (2009). The morality of harm. Cognition, 113(1), 80-92. https://doi.org/10.1016/j.cognition.2009.06.015

Stieger, S., \& Reips, U. D. (2016). A limitation of the Cognitive Reflection Test: familiarity. PeerJ, 4, e2395. https://doi.org/10.7717/peerj.2395

Ten Have, T. R., Normand, S. L. T., Marcus, S. M., Brown, C. H., Lavori, P., \& Duan, N. (2008). Intent-to-treat vs. non-intent-to-treat analyses under treatment non-adherence in mental health randomized trials. Psychiatric annals, 38(12). https://doi.org/10.3928/0048571320081201-10

Tepe, B., \& Aydinli-Karakulak, A. (2019). Beyond harmfulness and impurity: Moral wrongness as a violation of relational motivations. Journal of personality and social psychology. https://doi.org/10.1037/pspi0000169

Turiel, E. (1983). The development of social knowledge: Morality and convention. Cambridge: Cambridge University Press.

Tybur, J. M., Lieberman, D., \& Griskevicius, V. (2009). Microbes, mating, and morality: individual differences in three functional domains of disgust. Journal of personality and social psychology, 97(1), 103. https://doi.org/10.1037/a0015474

Uhlmann, E. L., Pizarro, D. A., \& Diermeier, D. (2015). A person-centered approach to moral judgment. Perspectives on Psychological Science, 10(1), 72-81.

https://doi.org/10.1177/1745691614556679

\section{Acknowledgements}

This project was partially funded by the Research Division of the Universidad Nacional de Colombia, Bogotá, project number 37159/2017-2018. We thank the students involved in this project, and especially Gustavo Silva for his assistance with data collection. We are grateful to Marc Jekel, Joshua Knobe, and Andrew Monroe for very helpful comments on previous versions of this manuscript. 